\title{
Kinematic model inversions of hot star recurrent DAC data - tests against dynamical CIR models
}

\author{
J. Krtička ${ }^{1,2,3}$, R. K. Barrett ${ }^{1}$, J. C. Brown ${ }^{1}$, and S. P. Owocki ${ }^{1,4}$ \\ 1 Department of Physics and Astronomy, University of Glasgow, Glasgow G12 8QQ, UK \\ 2 Ústav teoretické fyziky a astrofyziky PřF MU, 61137 Brno, Czech Republic \\ 3 Astronomický ústav, Akademie věd České republiky, 25165 Ondřejov, Czech Republic \\ ${ }^{4}$ Bartol Research Institute, University of Delaware, Newark, DE 19716, USA
}

Received 22 September 2003 / Accepted 21 December 2003

\begin{abstract}
The Discrete Absorption Components (DACs) commonly observed in the ultraviolet lines of hot stars have previously been modelled by dynamical simulations of Corotating Interaction Regions (CIRs) in their line-driven stellar winds. Here we apply the kinematic DAC inversion method of Brown et al. to the hydrodynamical CIR models and test the reliability of the results obtained. We conclude that the inversion method is able to recover valuable information on the velocity structure of the mean wind and to trace movement of velocity plateaux in the hydrodynamical data, though the recovered density profile of the stream is correct only very near to the stellar surface.
\end{abstract}

Key words. stars: early-type - stars: winds, outflows - stars: mass-loss - line: profiles

\section{Introduction}

The time-averaged structure of line-driven winds of hot $\mathrm{OB}$ stars can be adequately described by time-independent wind models (e.g. Hillier \& Miller 1998; Vink et al. 2000; Pauldrach et al. 2001). However, winds of hot stars are well-known for the variability manifested in different domains of the electromagnetic spectrum. One of the most striking features in the spectra of hot star winds is the presence of Discrete Absorption Components (DACs). These DACs are superimposed on the (unsaturated) absorption part of P Cygni profiles and move from the lower blue-shift to higher blue-shift part of P Cygni profiles (e.g. Prinja \& Howarth 1988; Massa et al. 1995; Kaper et al. 1997; Prinja et al. 2002).

The main DAC features, i.e. their recurrence and slow acceleration, can be explained by the radiatively driven hydrodynamical simulations of Cranmer \& Owocki (1996). They assumed that DACs arise from Corotating Interaction Regions (CIRs), as first suggested by Mullan (1984). The origin of CIR formation is not yet known, though wind modulations by stellar pulsations or magnetic fields are often cited as possible drivers (Kaper et al. 1997; de Jong et al. 2001). Thus it is worthwhile to study the DAC phenomenon from data diagnostically, independent of any underlying driver model. A first step in this direction was made by Brown et al. (2004), who developed a kinematic data inversion method for the inference of absorbing stream properties from data on recurrent DACs.

Send offprint requests to: J. Krtička,

e-mail: krticka@physics.muni.cz
The optical depth variations calculated using the hydrodynamical structures of the Cranmer \& Owocki (1996) models on the other hand arise mainly due to the azimuthally localized velocity plateau (so-called Abbott kinks) propagating upstream in the stellar wind. These velocity plateaux are caused by the presence of radiative-acoustic waves (the so-called Abbott waves, Abbott 1980) moving with velocity higher than the sound speed. Thus, it is interesting to study how well the kinematic inversion method for line-profiles performs using the profiles from hydrodynamical CIR model calculations, specifically to see what the method recovers in terms of density and velocity variations.

\section{Kinematic inversion formulation}

The basic method of the DAC data inversion of Brown et al. (2004) can be summarised as follows. They assume that DACs arise as the result of an azimuthally varying mass-loss rate at the stellar surface, giving a density pattern in the wind that also varies with azimuth, and is then transported through the wind along the streamlines of an axisymmetric velocity law $v_{\mathrm{r}}=v_{\mathrm{r}}(r)$. The assumed axisymmetry of the wind velocity has several consequences:

1. From mass continuity it follows that the radial mass flux $n v_{\mathrm{r}}$ falls off with radius along any streamline as $1 / r^{2}$, which is the purely geometrical factor expected for essentially spherical wind expansion (which we view only in the equatorial plane - see below). 
2. From 1, it further follows that the density itself at any point along a streamline is related to the mass flux $n_{0} v_{0}$ at the point on the surface from which that streamline emanates by

$n(r)=\frac{v_{0} R^{2}}{v_{\mathrm{r}} r^{2}} n_{0} \quad$ (along a streamline),

where $R$ is the stellar radius.

3. The streamlines from any two points on the surface (at $\phi_{1}$ and $\phi_{2}$, say) will always have identical shapes for an axisymmetric velocity law, but will be shifted relatively in azimuth by $\phi_{2}-\phi_{1}$ at all radii. This means that the density pattern introduced into the wind at the surface will be preserved at all radii, but will be shifted in $\phi$ by the change in azimuth $\Delta \phi(r)$ of the streamlines due to rotation. In the corotating frame this is given by

$\Delta \phi(r)=\int_{R}^{r}\left[\Omega_{0}-\Omega(r)\right] \frac{\mathrm{d} r}{v_{\mathrm{r}}(r)}$,

where $\Omega(r) r=v_{\phi}$ is the rotational speed in the static frame and $\Omega_{0}=\Omega(R)$ is the angular velocity of the surface. (The density will also be scaled according to Eq. (1) along the streamlines.)

Putting these points together we can relate the physical density $n(r, \phi)$ of the wind at any point to the surface density profile $n_{0}(\phi)=n(R, \phi)$ by

$n(r, \phi)=\frac{v_{0} R^{2}}{v_{\mathrm{r}} r^{2}} n_{0}[\phi-\Delta \phi(r)]$,

so that the optical depth variation at the velocity $v=v_{\mathrm{r}}(r)$ corresponding to this radius is (from $\tau=n / v_{\mathrm{r}}^{\prime}$, in suitable units)

$\tau\left(v_{\mathrm{r}}, \phi\right) \equiv n(r, \phi) / v_{\mathrm{r}}^{\prime}=\mathcal{P}\left(v_{\mathrm{r}}\right) n_{0}(\phi-\Delta \phi(r))$,

where

$\mathcal{P}\left(v_{\mathrm{r}}\right)=\frac{v_{0} R^{2}}{v_{\mathrm{r}} v_{\mathrm{r}}^{\prime} r^{2}}$

gives the overall ( $\phi$-independent) weighting of the optical depth at each velocity and $v_{\mathrm{r}}^{\prime}$ is the velocity gradient.

The inversion method of Brown et al. (2004) uses the variation of the observed optical depth (using the Sobolev approximation Eq. (4)) with time (i.e., with observer azimuth, $\phi=\Omega_{0} t$ ) to determine the optical depth weighting function $\mathcal{P}\left(v_{\mathrm{r}}\right)$ and the spiral law $\Delta \phi(r)$, which then give the wind velocity law $v_{\mathrm{r}}(r)$ and rotation law $\Omega(r)$. Finally, from the known optical depth variations with azimuth at fixed radius (i.e., velocity) it is possible to calculate the surface density profile $n_{0}\left(\phi_{0}\right)$.

When the observed dynamical spectrum is consistent with the assumption of an axisymmetric velocity law it is easy to determine $\mathcal{P}(v)$ and $\Delta \phi(r)$. Integration of Eq. (4) over $\phi$ at fixed $v_{\mathrm{r}}$ gives

$\mathcal{P}(v) \propto \int_{0}^{2 \pi} \tau(v, \phi) \mathrm{d} \phi$.

Alternatively, if it is possible to identify the path of a single matter stream in the $\left(v_{\mathrm{r}}, \phi\right)$-plane, such as the peak of the density pattern in azimuth at each $v_{\mathrm{r}}$, the optical depth variation along this matter stream can be used to define $\mathcal{P}$, although this is likely to be more sensitive to data errors on the dynamical spectrum. To obtain $\Delta \phi(r)$ it is necessary to identify just such a matter stream, and to define its path in the $\left(v_{\mathrm{r}}, \phi\right)$ plane.

\section{Dynamical DAC prediction and kinematic model inversions}

\subsection{DAC prediction}

For the hydrodynamical prediction of DACs we apply a similar method to Cranmer \& Owocki (1996). We assume that DAC features in the spectra of OB stars are caused by CIRs in the stellar wind induced by a hot spot on the stellar surface. Although CIR formation in a radiatively driven wind is inherently three-dimensional, to make the problem more tractable we confine the hydrodynamical calculations only to the equatorial plane, $\theta=\pi / 2$.

In order to obtain static DAC models we relax the timedependent hydrodynamical equations to a steady-state. For the solution of time-dependent hydrodynamical equations we use the code VH-1 developed by J. M. Blondin and collaborators. The equations solved are equation of continuity (conservation of mass) in the form

$\frac{\partial \rho}{\partial t}+\frac{1}{r^{2}} \frac{\partial}{\partial r}\left(\rho v_{\mathrm{r}} r^{2}\right)+\frac{1}{r \sin \theta} \frac{\partial}{\partial \phi}\left(\rho v_{\phi}\right)=0$,

and the conservation of radial $(r)$ and azimuthal $(\phi)$ components of momentum,

$\frac{\partial v_{\mathrm{r}}}{\partial t}+v_{\mathrm{r}} \frac{\partial v_{\mathrm{r}}}{\partial r}+\frac{v_{\phi}}{r \sin \theta} \frac{\partial v_{\mathrm{r}}}{\partial \phi}=\frac{v_{\phi}^{2}}{r}-\frac{1}{\rho} \frac{\partial P}{\partial r}+g_{\mathrm{eff}}+g^{\text {lines }}$,

$\frac{\partial v_{\phi}}{\partial t}+v_{\mathrm{r}} \frac{\partial v_{\phi}}{\partial r}+\frac{v_{\phi}}{r \sin \theta} \frac{\partial v_{\phi}}{\partial \phi}=-\frac{v_{\mathrm{r}} v_{\phi}}{r}-\frac{1}{\rho r \sin \theta} \frac{\partial P}{\partial \phi}$,

where $\rho$ is the local wind density, $v_{\mathrm{r}}$ and $v_{\phi}$ are the radial and azimuthal components of wind velocity, $t$ is time, and gas pressure $P$ is evaluated using the perfect gas equation of state.

The effective gravitational acceleration is given by

$g_{\mathrm{eff}}=-\frac{G M(1-\Gamma)}{r^{2}}$,

where $G$ is the gravitational constant, $M$ is the stellar mass and the Eddington factor $\Gamma$ accounts for the radiative force due to electron scattering. The radiative acceleration due to lines in the Castor et al. (1975) approximation is given by

$g^{\text {lines }}=k f A(\psi) \frac{G M \Gamma}{r^{2}}\left(\frac{n_{\mathrm{e}} / W}{10^{11} \mathrm{~cm}^{-3}}\right)^{\delta}\left(\frac{1}{\kappa_{\mathrm{e}} v_{\mathrm{th}} \rho}\left|\frac{\partial v_{\mathrm{r}}}{\partial r}\right|\right)^{\alpha}$,

where $k, \alpha$ and $\delta$ are line-force parameters (Abbott 1982; Puls et al. 2000), $f$ is the finite-disk correction factor (Friend \& Abbott 1986; Pauldrach et al. 1986), $n_{\mathrm{e}}$ is the electron number density, $W$ is the dilution factor, $\kappa_{\mathrm{e}}$ is the opacity due to the electron scattering, and $v_{\text {th }}$ is a fiducial ion thermal speed (by definition hydrogenic). The function $A(\psi)$ accounts for the force enhancement due to the spot, where the corotating azimuthal angle $\psi$ is given by

$\psi=\phi-\Omega_{0} t$, 
with the star's rotational angular velocity $\Omega_{0}=V_{\text {rot }} / R_{*}$. The force amplitude has the form

$A(\psi)=1+A_{0} \exp \left[-\left(\psi-\psi_{0}\right)^{2} / \sigma^{2}\right]$,

where $A_{0}$ is the dimensionless spot amplitude (corresponding to the flux enhancement), $\psi_{0}$ is the corotating azimuthal position of the center of the spot and $\sigma$ describes the spatial extent of the spot on the star. Thus, contrary to the paper of Cranmer \& Owocki (1996), we have neglected limb-darkening.

The flow variables are specified on a fixed two-dimensional mesh in radius and azimuth. We use 250 radial zones, extending from $r=R_{*}$ to $r=11 R_{*}$ with higher concentration of zones near the stellar base. The spatial zone width radially increases by $1 \%$ per zone. The azimuthal mesh contains 180 constantly spaced zones, ranging from 0 to $2 \pi$. The boundary conditions are specified in the same way as in Owocki et al. (1994) and Cranmer \& Owocki (1996). As an initial condition we use the relaxed one-dimensional model of Krtička \& Kubát (2001).

The DAC profile is calculated using the solution of the radiative transfer equation in the absence of any source function term in a point-star approximation as

$\tau(v, \phi)=\int_{R_{*}}^{\infty} \kappa \rho(r, \phi) \exp \left[-\left(v_{\mathrm{r}}(r, \phi)-v\right)^{2} / v_{\mathrm{G}}^{2}\right] \mathrm{d} r$,

where $\kappa$ is line-opacity (its arbitrary value is selected to obtain optical depths of order unity) and $v_{\mathrm{G}}$ descibes profile broadening as a sum of temperature broadening and microturbulent broadening by

$v_{\mathrm{G}}^{2}=v_{\mathrm{th}}^{2}+v_{\mathrm{turb}}^{2}$,

where $v_{\text {turb }}$ is a microturbulent velocity.

Surprisingly, the moving absorption features (DACs) in the spectra calculated from the hydrodynamical simulations are not predominantly caused by density variations. This can be seen from the fact that the wind optical depth can be approximated by the so-called Sobolev approximation in the form of

$\tau \sim \frac{\rho}{v_{\mathrm{r}}^{\prime}}$.

Even with relatively small variations of wind density and velocity the variations of velocity gradient and also of the Sobolev optical depth may be large. This is especially the case when a typical feature of our hydrodynamical calculations develops, the so-called Abbott kink. In the region of an Abbott kink the velocity derivative changes its sign from positive to negative and back. Thus, as can be seen from Eq. (16), the optical depth variations due to the Abbott kink may be huge.

\subsection{Kinematic model inversion}

For the inversion of the DAC data (calculated using hydrodynamical simulations) we use essentially the same code as Brown et al. (2004). The DAC data velocities are normalised with respect to the maximal velocity obtained in the hydrodynamical simulations, and only optical depth variation data for lower velocities are used in the kinematic model inversion procedure. Finally, for the inversion of the velocity law, we take into account that the DAC profiles are calculated using data from only finite intervals of radius.

\section{Results}

For our simulated study we selected the parameters of the star HD 64760 because its wind variability was extensively studied in the course of the IUE "MEGA" campaign (Prinja et al. 1995). Stellar and wind parameters used for the hydrodynamical simulations are given in Table 1. Stellar parameters were taken from Hoffleit \& Jaschek (1982), Humphreys \& McElroy (1984), and Schaller et al. (1992, see Fullerton et al. 1997) and for the parametrization of radiative force we used generic parameters obtained by Pauldrach et al. (1990). The value of the microturbulent velocity was selected to obtain smooth wind profiles. Its value does not much influence the results obtained.

Comparisons of data from hydrodynamical simulations and from inverted data are given in Figs. 1-3 for spots with relative amplitudes $A=0.05,0.2,0.5$. We will discuss the reliability of each inverted relation separately.

Table 1. Stellar and wind parameters of HD 64760.

\begin{tabular}{cc}
\hline \hline Parameter & Value \\
\hline$R_{*}$ & $22 R_{\odot}$ \\
$V_{\text {rot }}$ & $238 \mathrm{~km} \mathrm{~s}^{-1}$ \\
$T_{\text {eff }}$ & $23100 \mathrm{~K}$ \\
$M$ & $20 M_{\odot}$ \\
$L$ & $1.2 \times 10^{5} L_{\odot}$ \\
$\alpha$ & 0.650 \\
$k$ & 0.050 \\
$\delta$ & 0.28 \\
$v_{\text {turb }}$ & $50 \mathrm{~km} \mathrm{~s}^{-1}$ \\
\hline
\end{tabular}

Apparently, the inversion of the mean velocity law $v_{\mathrm{r}}(r)$ is very good (middle left panels). Clearly, because during the inversion process we assumed that the radial wind velocity is independent of azimuth, it cannot describe the azimuthal variations of wind velocity caused by the outward motion of an Abbott kink. However, it is possible to obtain a very good inference of the $\phi$-averaged velocity law, at least for low-amplitude perturbations. The main reason is that the inversion method used is able to find the velocity law even for a nonvariable wind via the inversion of the unperturbed absorption profile.

On the other hand the interpretation of the inverted rotational law is more complicated. The optical depth variations of the line profiles calculated using hydrodynamical simulations are caused mainly by the velocity variations due to the Abbott kink. Thus, we might expect that the inversion method would trace the movement of the Abbott kink and not the movement of real fluid parcels. To test this consideration, we calculated the radial variation of the azimuth of onset for the Abbott kink relative to the stellar spot. We define the onset of the Abbott kink as the point with the minimal radius where $\partial v_{\mathrm{r}} / \partial r<0$. We have compared the radial variations of the azimuth of the onset for the Abbott kink and of the azimuth of the fluid parcel (calculated for constant $\phi=0.4$; results for other other azimuths are very similar) with the radial variation infered from inversion. These results are also plotted in the upper-right panels of Figs. 1-3. Inspecting Figs. 1-3 we conclude that the "density" locus $\Delta \phi(r)$ found by the inversion method in reality always 

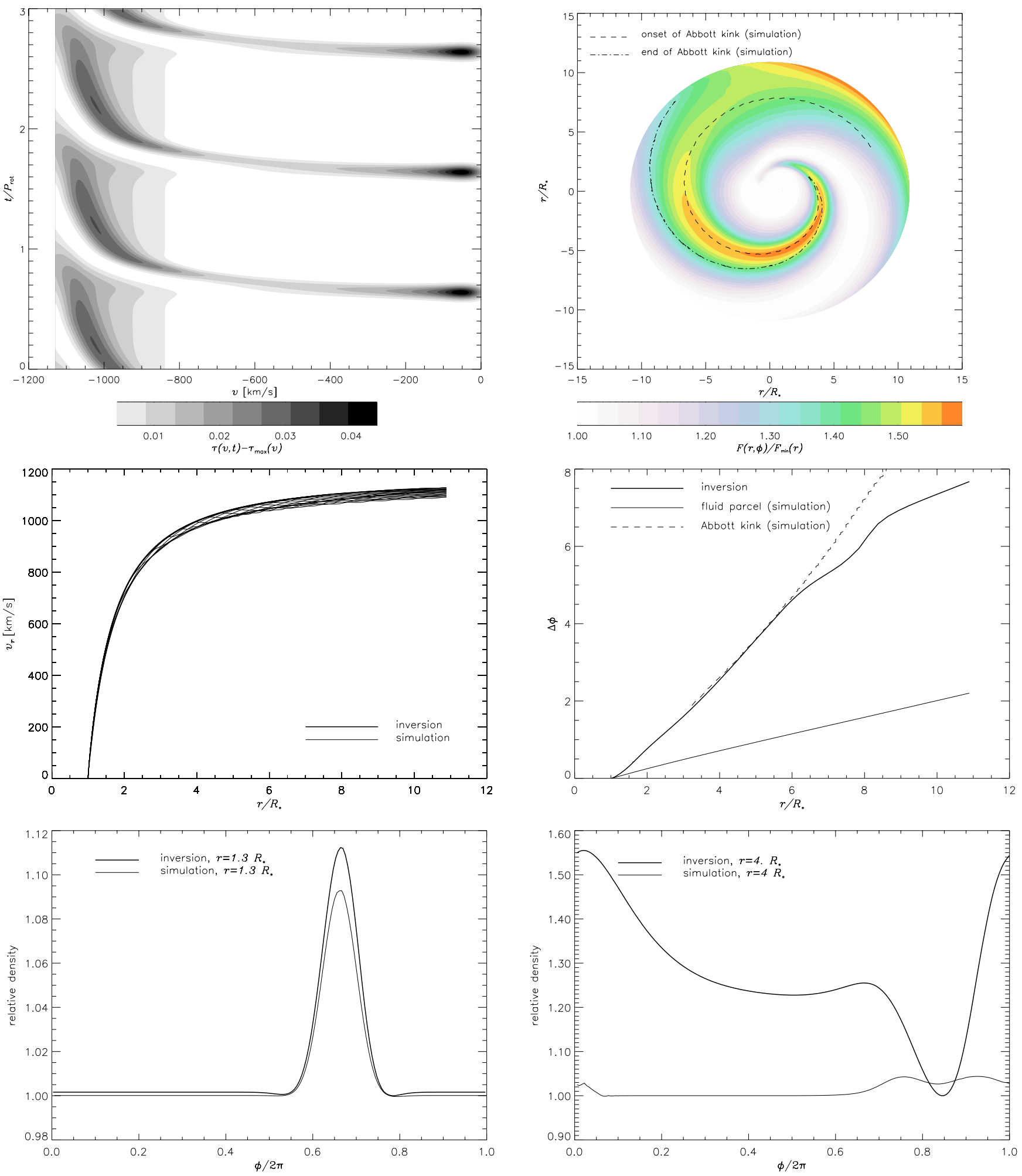

Fig. 1. Comparison of hydrodynamical simulations and inverted data for a spot with relative amplitude $A=0.05$. Upper left panel: variation of DAC optical depth (calculated as a difference to minimal absorption template) derived using the data from simulations. Upper right panel: inverted variations of effective density and the locations of the onset and end of Abbott kink. Middle left panel: radial velocity $v_{\mathrm{r}}(r, \phi)$ taken from simulations at 18 equally spaced azimuths and the inverted mean radial velocity $v_{\mathrm{r}}(r)$. The Abbott kink in the hydrodynamical calculations can be clearly recognized. Middle right panel: radial variation of azimuths $\Delta \phi$ of a fluid parcel relative to the spot, inverted location of density peak in azimuth and the azimuth of the onset of Abbott kink. Lower panels: variation of density from the simulations $\rho(r, \phi)$ relative to the the density of $1 \mathrm{D}$ flow without a spot $\rho_{1 \mathrm{D}}(r)$ and inverted density variation $F(r, \phi)$ for two different radii. 

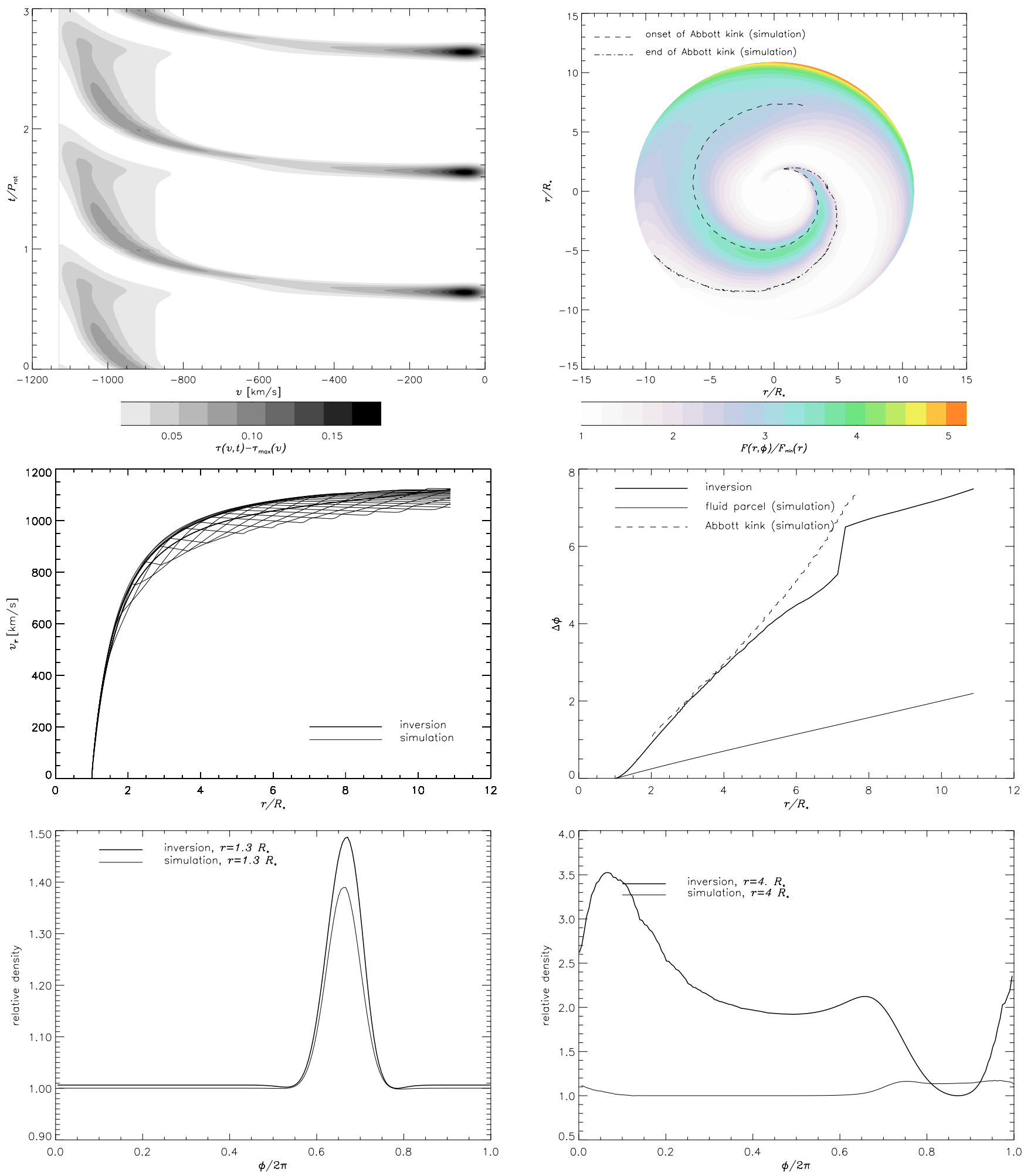

Fig. 2. The same as Fig. 1, however for spot with relative amplitude $A=0.2$.

traces the location of an Abbott kink and in fact does so with a high degree of reliability in those parts of the stellar wind where the Abbott kink is already formed.

We plotted also (lower panels of Figs. 1-3) the relative azimuthal variation of the relative CIR density profile $F(r, \phi)$ obtained using the kinematic inversion method and the actual density profile calculated using hydrodynamical simulations (plotted relatively to the unperturbed wind model). Their comparison shows that the inversion method is able to infer density profiles well only relatively near to the stellar surface, where the optical depth variations are not yet dominated by the variations of the velocity gradient. This is not the case in the outer parts of the wind where both density profiles are clearly different. This is caused by the formation of the Abbott kink, which 

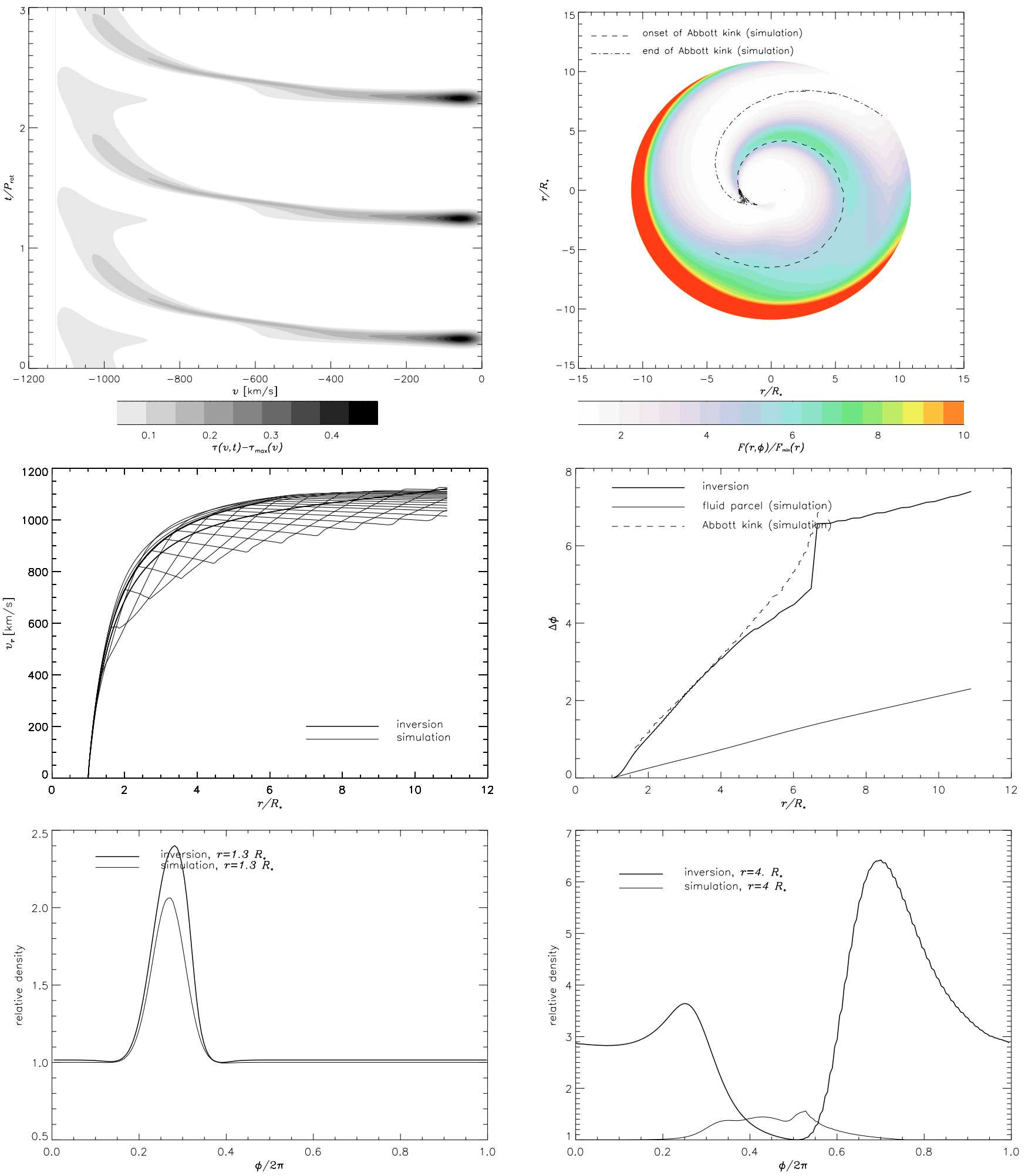

Fig. 3. The same as Fig. 1, however for spot with relative amplitude $A=0.5$.

enhances the optical depth. Note however that the spot azimuth and its width at the stellar surface can be relatively well inferred from the kinematic inversion method.

The fact that the main contribution to the DAC optical depth is given by the moving Abbott kink also does not allow us to invert to get correct wind densities. As discussed in Sect. 2 the original method of Brown et al. (2004) assumes that the relative variations of wind density profile in azimuth are the same throughout the stellar wind. This also imposes limitations on the azimuthal variations of DAC optical depths. The situation is different with the azimuthal optical depth variations calculated with the data from simulations, since the main contribution to the DAC optical depth in this case is given by the moving Abbott kink. However, it is possible to slightly modify 
the original Brown et al. (2004) method to obtain the effective wind density that produces the DAC from simulations. Clearly, this effective density does not trace the real wind matter density, but rather the optical depth variations, which are mainly given by the variation of velocity gradient due to the Abbott kink (see Eq. (16)). This situation is demonstrated in lower panels of Figs. 1-3 where azimuthal density variations from simulations and from inversion are compared. Apparently, relatively near the stellar surface, the variations of velocity gradient are lower, and the optical depth variations are influenced mostly by density variations. Thus, it is possible to infer relatively well the correct location of a footpoint of a spot at the stellar surface and its geometrical width. However, in the outer parts of the stellar wind, where the Abbott kink is already formed, the contribution of velocity gradient variations to the optical depth variations is dominant. Thus, the inversion method in fact traces the movement of the Abbott kink. This can be seen from upper left panels of Figs. 1-3 where density variations obtained from inversion are compared with the location of the onset and end of Abbott kink (defined as the radius where velocity gradient changes its sign from positive to negative and from negative to positive respectively).

\section{Discussion}

We applied the kinematic inversion method of Brown et al. (2004) to DAC profiles calculated using hydrodynamical CIR models. We compared CIR structure inferred by kinematic inversion with the actual simulated one, as an important test of reliability of the inversion method.

Although the kinematic inversion method is not able to calculate azimuthal variation of radial velocities, we showed that it gives reliable results for mean wind radial velocities even for relatively bright surface spots. However, because the highest optical depths of DACs in the numerical simulations arise mainly from spiralling velocity plateaux (Abbott kinks) and not from density enhancements, the inversion method does not trace the movement of real fluid parcels, but of an Abbott kink. Thus, it is possible from DAC data to trace the spiral law of Abbott kinks propagating in the perturbed stellar wind. Near the stellar surface the spiral law of these patterns is inferred with a high degree of accuracy. This is with quantitative accuracy and gives a general indication of where absorbing matter lies. Finally, it is not possible to invert to get a reliable density profile of the CIR, due to the above mentioned dependency of optical depth variations on the occurence of velocity plateaux, and due to the non-monotonic velocity law. Clearly, density variations are always overestimated. The density profile has the correct amplitude only very near to the stellar surface, where the velocity gradient variations do not significantly influence the optical depth. However, it is possible to obtain the correct azimuth of the CIR footpoint and its extent at the stellar surface.
Note that we do not require that the wind variability manifested by the presence of DACs in the wind profiles of hot stars be caused by hot spots at the stellar surface. The hot spot model provides merely a convenient base perturbation for the generation of CIRs. The DAC profile calculated using this CIR model predicts all basic properties of observed DACs. Thus, it provides an appropriate cornerstone for any technique aiming to infer real properties of the material causing DACs. We can easily test which properties of CIRs can be inferred from the observed DAC spectra and which cannot. We conclude that kinematic inversion method is able to infer some useful information on several properties of the material causing the DACs and thus can be potentially used for observational tests of future DAC theories.

Acknowledgements. This research has made use of NASA's Astrophysics Data System. This work was supported by a UK PPARC Rolling Grant, by Czech grants GA ČR 205/01/0656 and 205/02/0445. S.P.O. acknowledges support from a PPARC fellowship for sabbatical research in the UK, and from NSF grant AST00-97983 to the University of Delaware.

\section{References}

Abbott, D. C. 1980, ApJ, 242, 1183

Abbott, D. C. 1982, ApJ, 259, 282

Brown, J. C., Barrett, R. K., Owocki, S. P., et al. 2004, A\&A, 413, 959

Castor, J. I., Abbott, D. C., \& Klein, R. I. 1975, ApJ, 195, 157

Cranmer, S. R., \& Owocki, S. P. 1996, ApJ, 462, 469

Friend, D. B., \& Abbott, D. C. 1986, ApJ, 311, 701

Fullerton, A. W., Massa, D. L., Prinja, R. K., Owocki, S. P., \& Cranmer, S. R. 1997, A\&A, 327, 699

Hillier, D. J., \& Miller, D. L. 1998, ApJ, 496, 407

Hoffleit, D., \& Jaschek, C. 1982, The Bright Star Catalogue, 4th edition, Yale University Observatory, New Haven

Humphreys, R. M., \& McElroy, D. B. 1984, ApJ, 284, 565

de Jong, J. A., Henrichs, H. F., Kaper, L., et al. 2001, A\&A, 368, 601

Kaper, L., Henrichs, H. F., Fullerton, A. W., et al. 1997, A\&A, 327, 281

Krtička, J., \& Kubát, J. 2001, A\&A, 377, 175

Massa, D., Fullerton, A. W., Nichols, J. S., et al. 1995, ApJ, 452, L53

Mullan, D. J. 1984, ApJ, 283, 303

Owocki, S. P., Cranmer, S. R., \& Blondin, J. M. 1994, ApJ, 424, 887

Pauldrach, A. W. A., Hoffmann, T. L., \& Lennon, M. 2001, A\&A, 375, 161

Pauldrach, A. W. A., Kudritzki, R. P., Puls, J., \& Butler, K. 1990, A\&A, 228, 125

Pauldrach, A., Puls, J., \& Kudritzki, R. P. 1986, A\&A, 164, 86

Prinja, R. K., \& Howarth, I. D. 1988, MNRAS, 233, 123

Prinja, R. K., Massa, D., \& Fullerton, A. W. 1995, ApJ, 452, L61

Prinja, R. K., Massa, D., \& Fullerton, A. W. 2002, A\&A, 388, 587

Puls, J., Springmann, U., \& Lennon, M. 2000, A\&AS, 141, 23

Schaller, G., Schaerer, D., Meynet, G., \& Maeder, A. 1992, A\&AS, 96, 269

Vink, J. S., de Koter, A., \& Lamers, H. J. G. L. M. 2000, A\&A, 362, 295 